\title{
Azimuth-Switched Quantization for SAR Systems and Performance Analysis on TanDEM-X Data
}

\author{
Michele Martone, Benjamin Bräutigam, and Gerhard Krieger, Fellow, IEEE
}

\begin{abstract}
In synthetic aperture radar (SAR) applications, raw data quantization represents an aspect of primary importance, since the number of bits employed for radar signal digitization affects on the one hand the on-board memory consumption and the data volume to be transmitted to the ground, but also on the other hand the quality of the SAR images. In this paper, we introduce a novel azimuth-switched quantization (ASQ) technique, which allows the implementation of non-integer quantization rates in a new, efficient way. This grants higher flexibility in terms of performance design and resource allocation, without increasing the complexity and the computational load of the quantization scheme. The presented results were obtained in the frame of the TanDEM-X mission.
\end{abstract}

Index Terms-Synthetic aperture radar (SAR), SAR interferometry (InSAR), TanDEM-X mission, block adaptive quantization (BAQ), azimuth-switched quantization (ASQ).

\section{INTRODUCTION}

$\mathbf{I}$ $\mathrm{N}$ the context of scalar quantization techniques for SAR systems, one of the most widely recognized methods of raw data digitization is the block adaptive quantization (BAQ). BAQ is a lossy data reduction technique, i.e. the data reconstructed after decompression are not equal to the original input samples. BAQ performs a space-varying estimation of raw data statistics, which are calculated for each block (with fixed size) of input data. This information is employed to set the quantization decision levels that best match with the observed statistics [1], [2]. The compression rates are fixed to integer numbers of bits. Despite its simplicity, BAQ has shown itself as an efficient solution for spaceborne SAR systems, where a huge amount of on-board data, due to large bandwidths, high PRFs and multiple polarizations, need to be stored and then transmitted to the ground. In the last years, non-integer quantization rates have been implemented to allow a finer performance and resource optimization, by following the quantizer (commonly based on a BAQ scheme) with additional software/hardware blocks (as, e.g., Huffman coders) at a cost of increasing the overall system complexity [3], [4], [5]. Moreover, for such quantization schemes part of the optimization process is performed on board and, therefore, the amount of data required for an acquisition cannot be exactly calculated during mission planning.

The quantization noise is given by the sum of two contributions: the granular noise, introduced by the reduced number of output values within the supported range, and the overload noise, due to the clipping of input signal exceeding the supported range. For the present scenario, however, it has

The TanDEM-X project is partly funded by the German Federal Ministry for Economics and Technology (Förderkennzeichen $50 \mathrm{EE}$ 1035). been verified that saturation effects occur very seldom in the data [6], and can be reasonably neglected. This assumption allows to model the quantization effects on the recorded raw data signals (in presence of homogeneous targets, cf. Section III-C) as an additive white Gaussian noise source [7], [8]. The signal at the output of the quantizer can be expressed as

$$
y(t)=A x(t)+A n(t)+q(t),
$$

where $x(t)$ is the input signal, $n(t)$ is the receiver noise, $A$ is the scaling factor peculiar of the quantization process [8], and $q(t)$ is the quantization noise. The fewer bits are employed for data compression, the higher is the quantization noise power, which directly affects the SAR image quality.

This letter describes a novel azimuth-switched quantization (ASQ) technique, which provides the capability of synthesizing fractional quantization rates without impacting the complexity and computational load of the quantization scheme. The impact on SAR and interferometric product quality is evaluated, and it is shown that performance and resources can be dynamically scaled with a very fine discretization. The analyses provided in this letter have been carried out in the frame of the TanDEM-X mission. TanDEM-X (TerraSAR$X$ add-on for Digital Elevation Measurement) is the first operational spaceborne bistatic SAR system and comprises the twin satellites TerraSAR-X (TSX, launched in 2007), and TanDEM-X (TDX, launched in June 2010). The primary objective of the mission is the generation of a worldwide and consistent digital elevation model (DEM) in bistatic configuration with an unprecedented accuracy [7]. On TSX and TDX, the received backscattered signal is first digitized by an 8-bit

TABLE I

ASQ RATES $\left(\hat{r}_{A S Q}\right)$ AND CORRESPONDING AZIMUTH SEQUENCES $\left(S_{\hat{r}_{A S Q}}, N=10\right)$.

\begin{tabular}{|c|c|}
\hline $\begin{array}{c}\text { ASQ Rate } \\
\text { [bits/sample] }\end{array}$ & $\begin{array}{c}\text { Azimuth Sequence } \\
\text { [bits/sample] }\end{array}$ \\
\hline 2.1 & 2222222223 \\
\hline 2.2 & 2222322223 \\
\hline 2.3 & 2232232232 \\
\hline 2.4 & 2322323223 \\
\hline 2.5 & 2323232323 \\
\hline 2.6 & 2323232333 \\
\hline 2.7 & 2323332333 \\
\hline 2.8 & 2333323333 \\
\hline 2.9 & 2333333333 \\
\hline
\end{tabular}






Fig. 1. Workflow for ASQ implementation on a SAR system. Integer rates are switched along the azimuth direction during the data take. After SAR processing, the focused image shows performance equivalent to as if the raw data was acquired with an "average" of the original sequence. In the example, a fractional rate of 2.5 bits/sample is obtained as a result of switching between 2 bits/sample and 3 bits/sample, range line by range line.

analog-to-digital converter (ADC) and then further compressed by the block adaptive quantizer. Possible compression rates are $8: 2$ (from 8 to 2 bits/sample), 8:3, 8:4, 8:6 and 8:8, where the latter corresponds to BAQ bypass [8], [6]. By taking into account the limited downlink capacity of TanDEM$\mathrm{X}$, for operational DEM acquisitions, BAQ 8:3 and 8:2 for both satellites are foreseen, which grant at least two global acquisitions within the nominal mission duration [7]. The main drawback of employing constant and integer compression rates is the high difference between resource consumption (and the corresponding image performance), especially for long data acquisitions. The ASQ technique described here can overcome such limitations, allowing a finer optimization of memory resource and performance. Moreover, the amount of data to be stored and transmitted to the ground can be precisely calculated during mission planning. A first demonstration with TSX and TDX proves that ASQ can successfully be used for state-of-the-art SAR systems, and that an overall performance improvement can be obtained. In the next section the principle of ASQ is explained. In Section III, a performance analysis on TanDEM-X data is presented: the impact of ASQ on the noise equivalent sigma zero (NESZ), azimuth ambiguities and interferometric coherence is investigated in detail. The letter is concluded in Section IV with a short summary.

\section{The AzImuth-Switched Quantization (ASQ) PRINCIPLE}

During the data collection by the SAR system, integer compression rates are toggled along the azimuth direction, at every echo range pulse (within a range line the quantization rate is kept constant). After performing the azimuth focusing over one synthetic aperture, an equivalent non-integer quantization rate is synthesized, which results from an "average" of the different rates used. That is, highly flexible adaptation of SAR performance and calculation of memory resources are possible, just by exploiting the peculiar SAR acquisition concept. Indeed, only a simple quantizer with integer rates is required (the capability of changing acquisition parameters during a SAR data take is a feature normally made available by the present state of technology). In Figure 1 the ASQ workflow is depicted for the case of 2.5 bits/sample. During the acquisition, the quantization setting is switched between
2 and 3 bits/sample at every range line (left part of the figure). The image is then processed (central part), showing performance equivalent to the average of the compression rates used along azimuth. It is worth restating that such a fractional rate is not the one of the focused image, for which an always constant bit-rate is used (e.g., 8 or 16 bits/pixel). Instead, it refers to an equivalent compression rate employed for the raw data: the processed image shows a performance as if the raw data were acquired with the rate resulting from the average of the original sequence.

On TSX and TDX the BAQ rate can be set for each range line, to support different compression rates for, e.g., dual polarization data. Anyhow, one could think to further extend the presented technique, adapting the quantization settings for each block within one echo window. This capability allows even finer sampling in terms of performance and resource allocation and is being considered for the design of future SAR systems (as, for example, the FD-BAQ technique implemented for the Sentinel-1 mission [4]).

The compression rate can be theoretically changed at every pulse repetition interval (PRI). Anyway, the maximum number of range lines $\left(n_{r g, \max }\right)$ to be taken into account for determining the effective average quantization rate are those "lying" inside one synthetic aperture $\left(L_{s a}\right)$ :

$$
n_{\text {rg }, \text { max }}=\frac{T_{S A R}}{\mathrm{PRI}}=\frac{L_{s a}}{v_{\text {sat }}} \cdot \frac{1}{\mathrm{PRI}},
$$

where $v_{\text {sat }}$ is the satellite velocity and $T_{S A R}$ is the integration time. For the TanDEM-X mission (and typical spaceborne configurations), $v_{s a t}$ is of about $7 \mathrm{~km} / \mathrm{s}$, the synthetic aperture $L_{s a}$ is longer than $3 \mathrm{~km}$, and the pulse repetition frequency (PRF) is bigger than $2 \mathrm{kHz}$ (i.e. PRI $<0.5 \mathrm{~ms}$ ). The resulting $n_{r g}$, max is around 1000: in practice, with the presented technique, bit rates until a fraction of a thousandth of a bit can be effectively implemented. A given ASQ rate $\hat{r}_{A S Q}$ is synthesized by replicating along azimuth direction the appropriate sequence of integer BAQ rates of length $N$ whose average is just $\hat{r}_{A S Q}$

$$
S_{\hat{r}_{A S Q}}=\left[r_{1}, r_{2}, r_{3}, \ldots, r_{N}\right]: \hat{r}_{A S Q}=\frac{1}{N} \sum_{k=0}^{N-1} r_{k} .
$$

In principle, given a certain $\hat{r}_{A S Q}$, the corresponding $S_{\hat{r}_{A S Q}}$ 

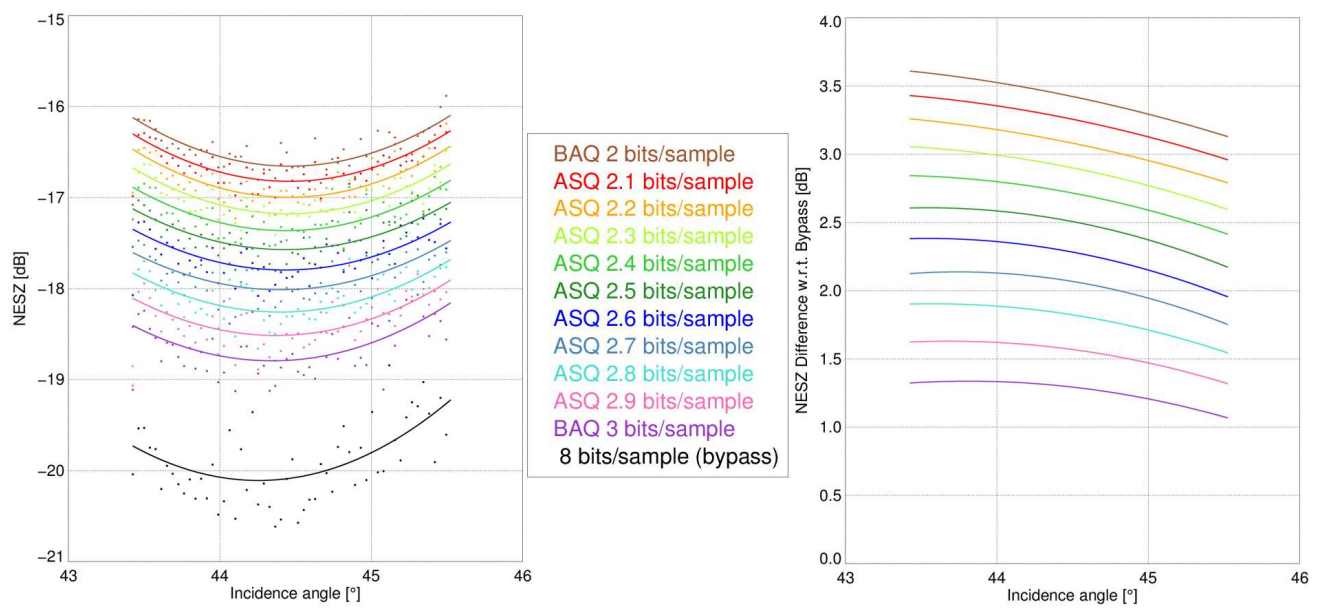

Fig. 2. (Left) NESZ for different ASQ rates, estimated from the rivers in the data take in Figure 3. The points show the recorded values from a distributed target analysis. By interpolating them, corresponding NESZ profiles are obtained, which are depicted with different colors. The highest and lowest curve indicate the NESZ for the case of BAQ 8:2 and BAQ bypass, respectively. (Right) NESZ degradation introduced by quantization with respect to the bypass case.

has to be as short as possible (i.e., $N=N_{\min }$ ), and the distribution of the rates needs to be homogeneous, in order to avoid biases in terms of performance loss within one synthetic aperture. To estimate quantization effects, raw data acquired with BAQ bypass (i.e., 8 bits/sample) have been recompressed on ground to different ASQ rates. These obtained products have been processed into SAR images, allowing the generation of interferograms, coherence maps and DEMs. The ASQ rates and the corresponding azimuth sequences used for the investigations presented in this letter are listed in Table I $(N=10)$ : each element of a sequence represents the compression rate employed for one range line. In the next section, the impact of ASQ on TanDEM-X interferometric and SAR performance is discussed and evaluated.

\section{ASQ PERformance ANALYsis}

In this section a performance analysis of ASQ on TanDEM$\mathrm{X}$ data is presented. The impact on the NESZ (III-A), azimuth ambiguities (III-B) and interferometric coherence (III-C) is investigated.

\section{A. Noise Equivalent Sigma Zero (NESZ)}

The signal-to-noise ratio (SNR) is a measure to describe the image quality of remote sensing systems, estimating how much a signal has been corrupted by noise (just as coherence does for interferograms). From a SAR image, the SNR is computed as follows:

$$
\mathrm{SNR}=\frac{\sigma_{0}}{\mathrm{NESZ}},
$$

where $\sigma_{0}$ is the backscatter coefficient and NESZ is the Noise Equivalent Sigma Zero, which accounts for the several noise sources affecting the data. For the present analysis, we may formally decompose the NESZ into the sum of two main contributions

$$
\mathrm{NESZ}=\mathrm{NESZ}_{\text {sys }}+\mathrm{NESZ}_{\text {quant }} .
$$

The first term on the right-hand side includes all error contributions induced by the system, like antenna pattern, instrument

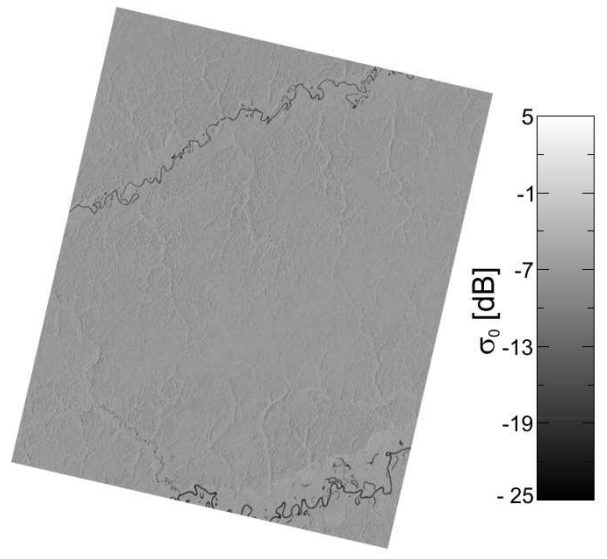

Fig. 3. Test area for NESZ estimation, located in Amazon rainforest (StripMap mode, HH polarization, acquired on June 21, 2012). NESZ profiles for different ASQ rates are estimated considering the backscatter response over the two rivers located on the upper-left and lower-right part of the scene.

thermal noise, as well as processing filters [7]. The second term describes the noise power solely due to quantization, which directly affects the radiometric resolution of the focused data. To estimate noise power from SAR images a distributed target analysis over regions showing very low backscatter response has been carried out. For our investigations, the area depicted in Figure 3 has been considered. It is located in the Amazon rainforest and is crossed by two rivers, entirely from near to far range: in particular, areas covered by flat water reflect almost completely the radar signal sent by the antenna to specular direction, and, therefore, the recorded signal is of the same order of magnitude as of the system noise (in this case, over the two rivers $\sigma_{0}<-19 \mathrm{~dB}$ ). After an averaging of the corresponding pixels along azimuth direction the NESZ for different ASQ rates could be evaluated. The results are depicted on the left-hand side of Figure 2. The dots indicate the measured values from a distributed target analysis. By interpolating along range direction, the resulting NESZ profile curves are derived (the integer rates have been obtained by 
using "pure" BAQ). On the right-hand side of Figure 2, the NESZ difference with respect to the bypass case is shown. Being all the acquisition and processing parameters the same except the ASQ rate employed, we can infer that the obtained profiles correspond just to the degradation term $\mathrm{NESZ}_{\text {quant }}$ in (5). As expected, a higher compression ratio corresponds to a higher degradation, varying between about $1 \mathrm{~dB}$ to $3.5 \mathrm{~dB}$ for the case of 3 bits/sample and 2 bits/sample, respectively.

\section{B. Azimuth Ambiguities}

Azimuth-switched quantization can be exploited to reduce the impact, among the others, of the azimuth ambiguities for a given data rate. In order to satisfy the sampling requirement, the PRF must be sufficiently high, depending mainly on the antenna flight velocity and on its azimuth length. In general, the higher the PRF, the smaller the azimuth-ambiguity-tosignal ratio (AASR). For a SAR system, the amount of data stored during an acquisition $\left(\right.$ Data $\left._{a c q}\right)$ is proportional to the data rate $r$ and the acquisition duration $\Delta t_{a c q}$

$$
D_{a t a} a c q \propto r \cdot \Delta t_{a c q} .
$$

The data rate is, in turn, proportional to the quantization rate $q_{\text {rate }}$ and the PRF, and often represents a bottleneck for spaceborne SAR systems. In order to evaluate the combined effect of quantization and PRF, simulations of a one-dimensional azimuth-compressed point target were carried out. In Figure 4, the peak AASR is depicted for BAQ rates between 2 bits/sample and 5 bits/sample and for different azimuth data rates $(8 \mathrm{kbits} / \mathrm{s}, 9 \mathrm{kbits} / \mathrm{s}$ and $10 \mathrm{kbits} / \mathrm{s}$; only one sample has been considered in range direction). The corresponding PRFs are in the range between $1500 \mathrm{~Hz}$ and $5000 \mathrm{~Hz}$. The fewer bits are employed for quantization (i.e., higher PRFs are allowed for the given data rate), the smaller AASR is observed. In particular, given a fixed data rate $\hat{r}$, a reduced ambiguous signal power can be obtained by reducing the quantization rate (i.e., moving to the left along one of the curves in Figure 4 and thus allowing an increased PRF). Alternatively, one can exploit a much finer-grained set in terms of acquisition parameters design and resource allocation in order to keep the ambiguous signal below a certain threshold (i.e., "jumping"



Fig. 4. Azimuth-ambiguity-to-signal ratio (AASR) over ASQ rates, for different data rates (depicted with different colors). For the described scenario, the resulting PRFs are in the range between $1500 \mathrm{~Hz}$ and $5000 \mathrm{~Hz}$. horizontally from one curve to another one). However, one has to be aware that for too high PRFs (depending on acquisition geometry and system parameters) it may happen that several successive pulses arrive at the receiving antenna at the same time. This produces artifacts in the SAR images known as range ambiguities. For the TanDEM-X case and the described scenario, anyway, a negligible worsening is expected in terms of range-ambiguity-to-signal ratio (RASR).

\section{Interferometric Coherence}

The key quantity to evaluate interferometric SAR (InSAR) performance is the coherence. It represents the normalized correlation coefficient between master (monostatic channel) and slave (bistatic channel) acquisition. Several error sources may contribute to coherence loss [7], [9]. On the left side of Figure 5 the interferometric coherence is shown for rates between 2 bits/sample and 3 bits/sample and for different test sites. For reference, performance resulting from uncompressed data is shown, too. Each curve describes the performance of one acquisition, which has been compressed to different ASQ rates. As expected, the fewer bits are employed for quantization, the lower coherence is obtained for the same test site. On the right side of the figure the coherence degradation with respect to the bypass case is depicted. The black bars represent the average and the standard deviation of the estimations for each ASQ rate, and good agreement with the theoretic prediction is observed, marked by the green lines for the case of two and three bits [7]. Moreover, it can be noticed that the dispersion of the estimations increases with a decreasing of the quantization rate. The reason for that is the different backscatter distribution in the analyzed scenes. Figure 6 shows the histograms for $\sigma_{0}$ of two areas with different backscatter characteristics. The salt lake of Uyuni (Bolivia), depicted in blue, is a quite homogeneous region, and the corresponding coherence loss (violet dots in Figure 5) goes from about 2\% to $8 \%$. On the other hand, the urban area of Las Vegas (USA), represented in red, shows a very inhomogeneous backscatter distribution, and a higher coherence degradation is observed (between 5\% and 14\%, marked in blue in Figure 5). This discrepancy in terms of performance is due to the so-called low scatterer suppression [10]: the responses from contiguous targets (with respect to the synthetic antenna and the chirp length) overlap considerably in the raw data domain. On the other hand, the decision levels as well as the clipping threshold for quantization process are set according to the mean power of the raw data block. Therefore, if two targets have different magnitude response, the quantizer matches best with the strong signal, whereas the low one is heavily distorted. Such an effect represents an additional nonlinear and signal-dependent error source in the SAR data and is currently being investigated for TanDEM-X.

TSX and TDX have a relatively limited downlink capacity, which is due to an average contact time with the ground station network of about $10 \mathrm{~min} /$ orbit at a total net data rate of about $260 \mathrm{Mbits} / \mathrm{s}$. If considering nominal acquisition parameters, this implies an allowed time for data takes of about $180 \mathrm{~s}$ per orbit, for the case of a quantization with 3 


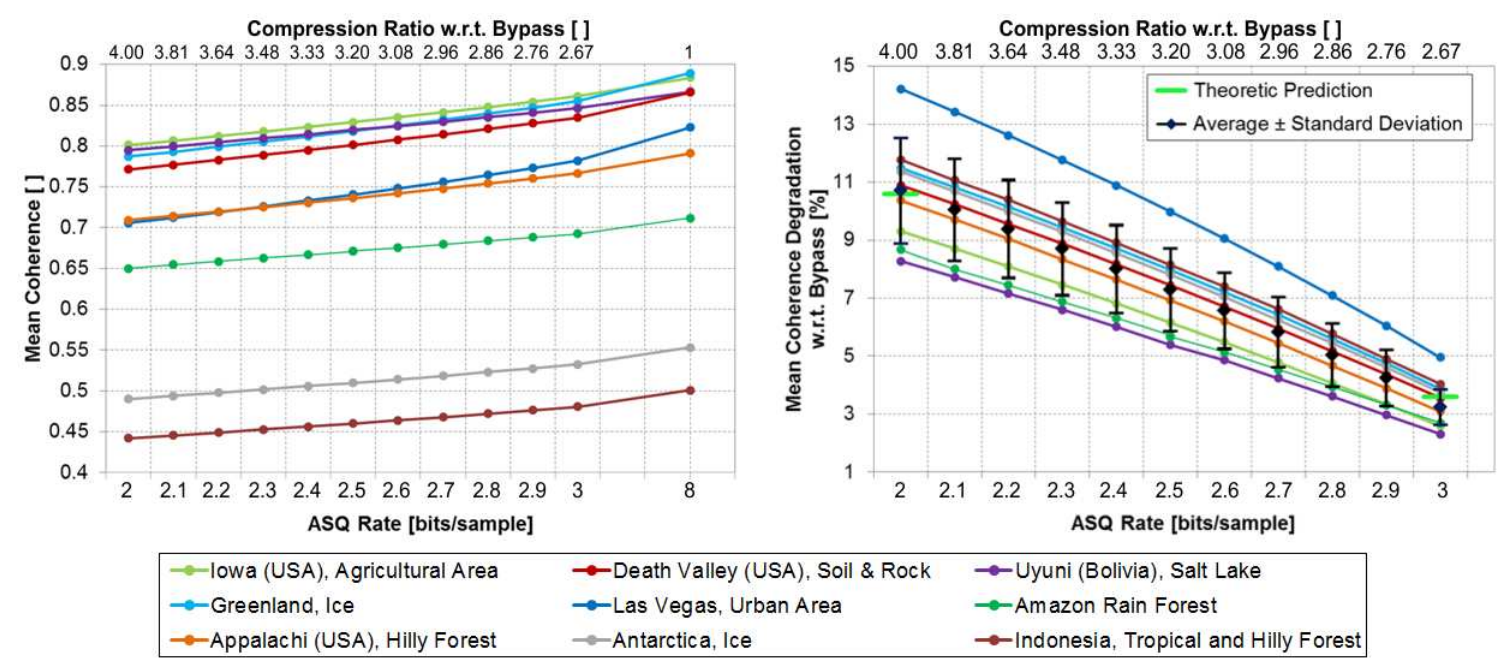

Fig. 5. (Left) Interferometric coherence over ASQ rates and (right) coherence degradation with respect to the bypass case, for different test sites (depicted with different colors). The black bars on the right represent the average and the standard deviation of the estimated degradations for each ASQ rate, and the short horizontal green lines indicate the theoretic prediction for the case of two and three bits [7]. Each curve corresponds to one acquisition at different ASQ rates. On the upper part of the charts, the compression ratio for each ASQ rate is specified.

bits/sample [7]. In order to speed up the completion of the global DEM acquisition, it has been considered the opportunity to further reduce the quantization rate: in particular, a "hybrid" quantization of $2.5 \mathrm{bits} / \mathrm{sample}$ has been exploited, using 3 bits/sample on one satellite and 2 bits/sample on the other, for the acquisition of areas showing very good performance and homogeneous backscatter response. It has been verified that the interferometric performance was essentially the same as that employing an ASQ rate of 2.5 bits/sample, and that a considerable increase, in terms of allowed acquisition time, of about $40 \mathrm{~s}$ per orbit (compared to a quantization with 3 bits/sample) could be obtained.

\section{CONClusions}

In this letter, we have presented a novel azimuth-switched quantization (ASQ) scheme for SAR raw data, which allows to implement non-integer compression rates in a new, efficient way. The principle of the proposed technique has been described and the impact on SAR and interferometric performance of TanDEM-X experimental data has been evaluated and quantified. It has been shown that highly flexible adaptation of SAR performance and calculation of memory consumption is achievable. This provides more freedom in the design and planning of SAR missions, when memory and downlink resources are limited.

\section{REFERENCES}

[1] D. Lancashire, B. Barnes, and S. Udall, "Block adaptive quantization," US Patent US 6255987, 07 3, 2001. [Online]. Available: http://www.patentlens.net/patentlens/patent/US6255987/

[2] R. Kwok and W. T. K. Johnson, "Block adaptive quantization of Magellan SAR data," IEEE Geosci. Remote Sens. Lett., vol. 27, no. 4, pp. 375-383, July 1989.

[3] T. Algra, "Data compression for operational SAR missions using entropy-constrained block adaptive quantization," in Proc. Int. Geosci. Remote Sens. Symp., Toronto, Canada, June 2002, pp. 1135-1138.

[4] E. Attema, C. Cafforio, M. Gottwald, P. Guccione, A. M. Guarnieri, F. Rocca, and P. Snoeij, "Flexible dynamic block adaptive quantization for Sentinel-1 SAR missions," IEEE Geosci. Remote Sens. Lett., vol. 7, no. 4 , pp. $766-770$, October 2010.

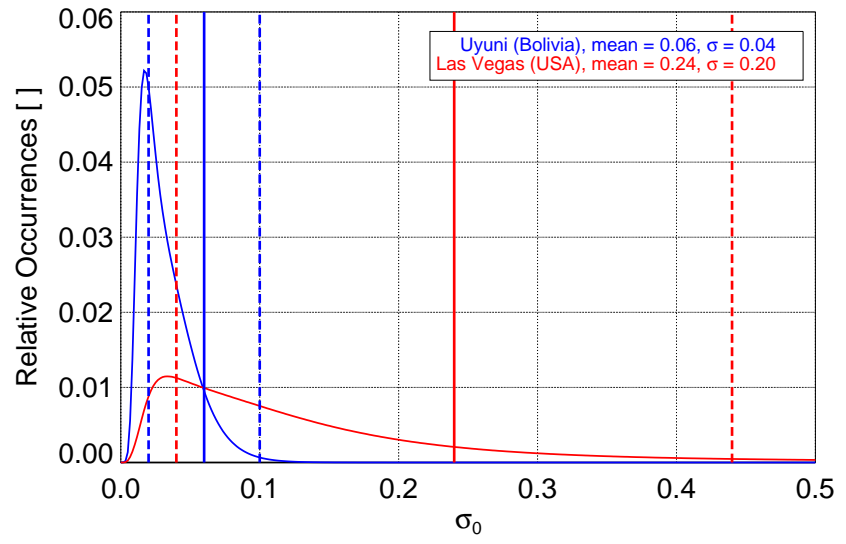

Fig. 6. Histogram for $\sigma_{0}$ measured over the salt lake of Uyuni (Bolivia), and the urban area of Las Vegas (USA). The continuous (dotted) lines indicate the mean (standard deviation) for each distribution. The linear scale highlights the difference in terms of dynamic range in backscatter.

[5] P. Guccione and A. M. Guarnieri, "A space adaptive quantizer for spaceborne SAR," IEEE Trans. Geosci. Remote Sens., vol. 49, no. 10, pp. 3564-3573, October 2011.

[6] J. Mittermayer, M. Younis, R. Metzig, S. Wollstadt, and J. MárquezMartínez, "TerraSAR-X system performance characterization and verification," IEEE Trans. Geosci. Remote Sens., vol. 48, no. 2, pp. 660-676, February 2010.

[7] G. Krieger, A. Moreira, H. Fiedler, I. Hajnsek, M. Werner, M. Younis, and M. Zink, "TanDEM-X: A satellite formation for high-resolution SAR interferometry," IEEE Trans. Geosci. Remote Sens., vol. 45, no. 11, pp. 3317-3341, November 2007.

[8] M. Younis, J. Böer, C. Ortega-Míguez, D. Schulze, S. Huber, and J. Mittermayer, "Determining the optimum compromise between SAR data compression and radiometric performance - an approach based on the analysis of TerraSAR-X data -," in Proc. Int. Geosci. Remote Sens. Symp., Boston, Massachussetts (USA), July 2008, pp. 107-110.

[9] M. Martone, B. Bräutigam, and G. Krieger, "Decorrelation effects in bistatic TanDEM-X data," in Proc. Int. Geosci. Remote Sens. Symp., Munich, Germany, July 2012, pp. 5558-5561.

[10] S. Huber, M. Younis, and G. Krieger, "The TanDEM-X mission: overview and interferometric performance," Int. J. Microw. Wireless Technol., vol. 2, no. 3-4, pp. 379-389, July 2010. 